\title{
Evaluation of radiological parameters after distal radius fracture in elderly people
}

\author{
Adam Zaręba \\ Department of Orthopedics and Trauma Surgery, General Hospital in Szczecinek, Poland
}

\begin{abstract}
Introduction. Distal radius fractures are one of the most frequent problems in every day practice and almost $17 \%$ of all fractures in adults. We have to very often face effects of low-energy trauma in elderly people. Osteoporotic bone and composed morphology of fracture forces surgeon to consider operation among the best options of treatment.

Aim. This is an attempt to compare radiological results achieved by closed reduction and cast immobilization versus open reduction and internal fixation with "Aptus ${ }^{\circledR ”}$ (Medartis, Basel Switzerland) volar plate, as well as dynamics of redisplacement during immobilization based on radiological parameters of distal radius.

Material and methods. There were 101 patients in examined group, aged 60 to 91.

Results. Internal fixation lead to smaller secondary displacement comparing to cast immobilization, but significancy varies depending on fracture morphology. Redisplacement during cast immobilization was comparable during first seven days and following weeks previous to removal of splint.
\end{abstract}

Keywords: distal radius, fracture, elderly, operative treatment.

\section{Introduction}

Distal radius fractures are one of the most frequent problems in every day practice and almost $17 \%$ of all fractures in adults [1]. We have to very often face effects of low-energy trauma in elderly people [2, 4]. Osteoporotic bone and composed morphology of fracture forces surgeon to consider operation among the best options of treatment.

Risk factors of distal radius fracture are osteoporosis, age, female gender, malnutrition as well as diseases leading to discoordination and causing greater risk of falling down [3]. There have been noticed greater occurrence during winter months [5].

Despite the high morbidity there is still no agreement when to choose more invasive methods in elderly people. Due to diametrically different biological condition of patients, from self sufficient to totally dependent, individual approach is needed. This is an attempt to compare results achieved by closed reduction and cast immobilization versus open reduction and internal fixation with "Aptus ${ }^{\circledR ”}$ (Medartis, Basel Switzerland) volar plate, as well as dynamics of redisplacement during immobilization based on radiological parameters of distal radius.

\section{Material and methods}

There were 101 patient after distal radius fracture in examined group, aged 60 to 91. 51 were treated operatively and 50 conservatively. Average age was respectively 69.9 and 72.8. In first group there were 49 woman and 2 men operated with "Aptus" volar plate. Indications for surgery were:

- palmar displacement

- dorsal displacement over $20^{\circ}$

- compression on dorsal cortex

- intraarticular fracture

- unsuccessful reposition

- redisplacement revealed in follow-up care.

Second group consisted of injured immobilized in the cast. It was satisfactory reposition, disqualification from surgery due to general condition or patient's per- 
sonal preferences that decided about assigning to this part. Patients with co-existing distal ulna fracture other than styloid process were excluded. Each time there was closed reposition performed to avoid soft tissue damage, as well as to decrease edema, pain and contracture. Morphology of fractures was classified accordingly to $\mathrm{AO}$ references (Figure 1, Table 1).

All patients underwent similar conservative treatment. In A\&E department hematoma block was performed by injecting approximately $15 \mathrm{cc}$ of lidocaine into fracture gap. Sedation with midazolam and fentanyl when required. Next reduction was performed using Sokolowski's apparatus, and after applying cast patients went for control X-Ray scan. Follow-up visits were scheduled for $7^{\text {th }}$ and $28^{\text {th }}$ day after fracture. If needed immobilization was prolonged for two additional weeks. Due to poor availability of rehabilitation no proper protocol could be used.

Patients qualified for operation were treated by open reduction and internal fixation with "Aptus" plate by Medartis. Two millimeter volar locking compression plate was used. Implant is made of titanium ASTM F136, what enables patient for MR examination, as well as reduces risk of immunologic reactions, infections and forming biofilm on its surface [6].

Approach was performed radially to flexor carpi radialis tendon, as described by Henry. It gives propper visualization of volar cortex, which is crucial for restoring radial length, and allows firm screws placement in subchondral bone. After fixation plate is cov- ered by pronator quadratus to prevent tendon irritation. Volar approach is first choice in instable fractures with volar displacement of distal fragment or if carpal tunnel decompression is needed. Appropriate implant localization is confirmed with image intensifier in P-A and lateral projections with additional radiocarpal joint scan. No drainage was performed. After pneumatic tourniquet was released few minute pressure was applied on the wound. Transverse carpal ligament was released only in patients with preoperative carpal tunnel syndrome $[8,9]$. After operation patients spent one or two days in the ward, when radiological control was performed and rehabilitation instructions given. In first 6 weeks only weight - free active and passive exercises were advised. Sutures were removed after 12 to 14 days. After 6 weeks and control X-ray scan patients were allowed to use hand freely (Figure 2, Table 2).

$X$-ray scan were assessed using calibrated measurement in "Exhibeon" 2.7. Four parameters were estimated - radial height, radial inclination, volar tilt and ulnar variance. In conservatively treated patients there was evaluated difference between the status after reposition and follow-up controls after 7 days and 4 to 6 weeks. In operated patients radiological assessment was conducted after surgery and after 6 weeks. Each parameter was measured twice to avoid measurement errors [10]. Obtained data was analyzed with Graph Pad Prism 5.1. Shapiro - Wilk's test revealed no normal distribution in both groups. Man - Whitney's was used to assess significancy of differences in both groups.

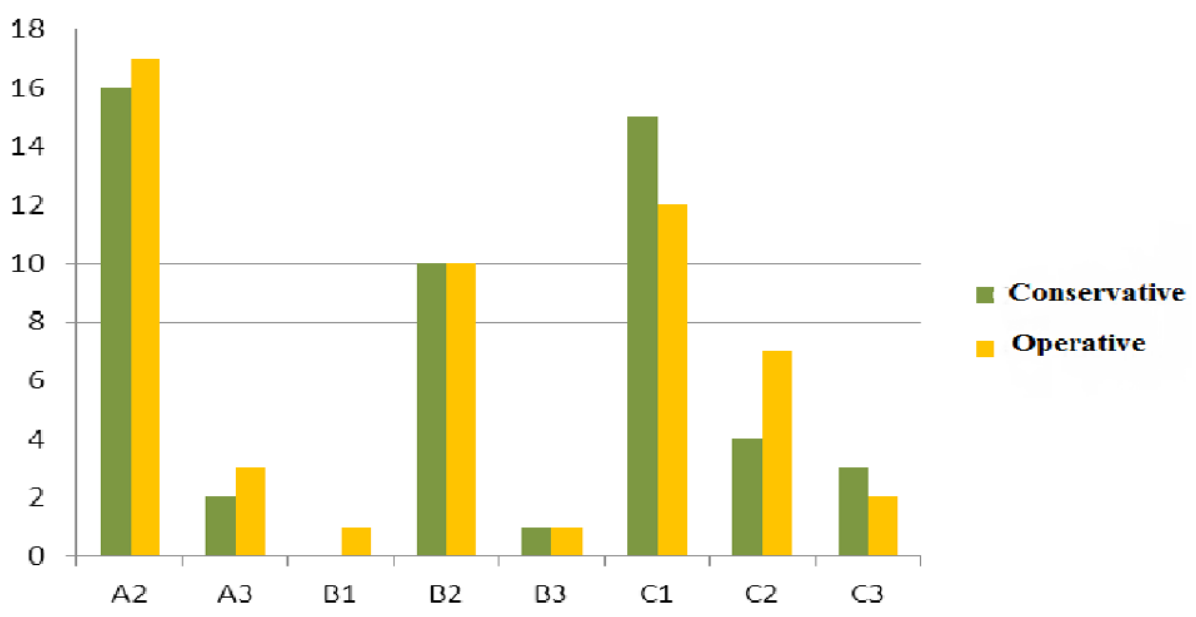

Figure 1. Distribution of fracture cases according to $\mathrm{AO}$ classification

Table 1. Distribution of fracture cases according to AO classification

\begin{tabular}{lcccccccc}
\hline & A2 & A3 & B1 & B2 & B3 & C1 & C2 & C3 \\
\hline Conservative & 16 & 2 & 0 & 10 & 1 & 15 & 4 & 3 \\
\hline Operative & 17 & 3 & 1 & 10 & 1 & 12 & 7 & 2 \\
\hline
\end{tabular}




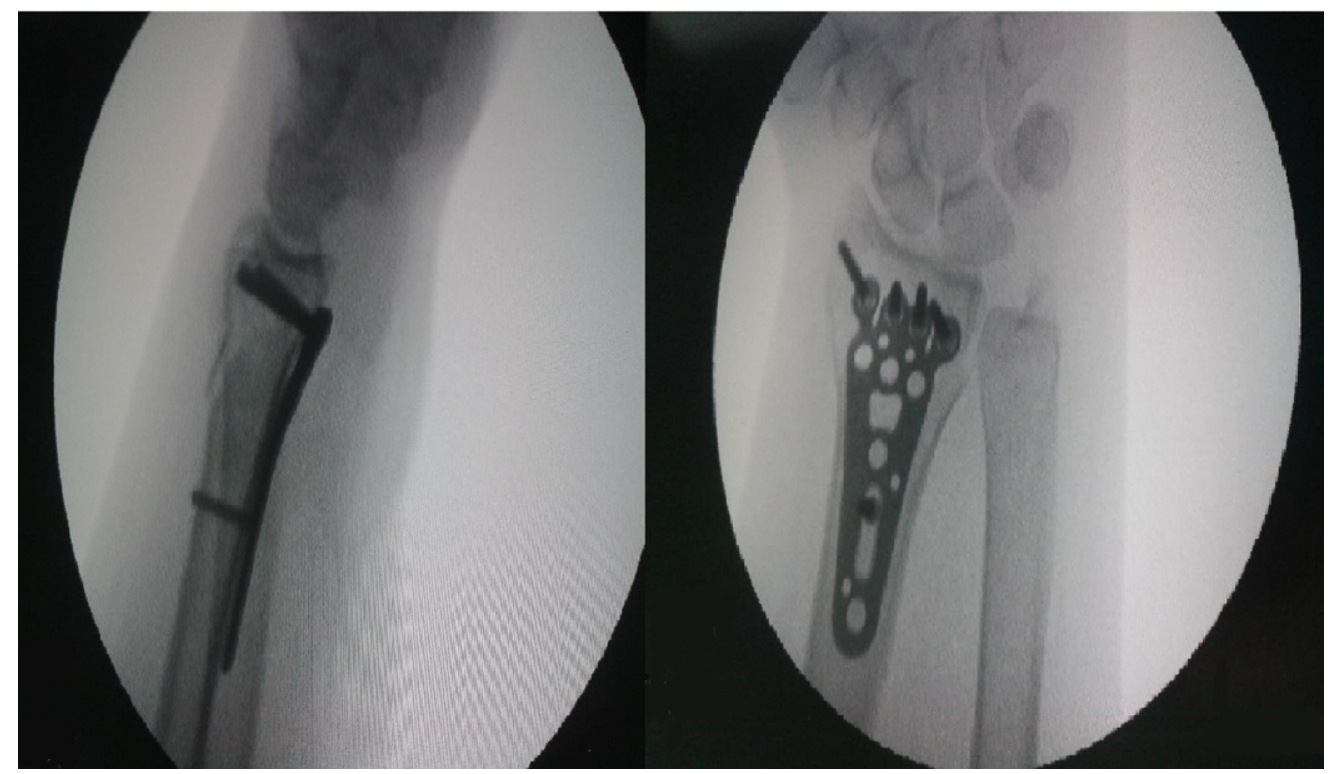

Figure 2. Confirmation of proper placement of the plate

Table 2. Average radiological parameters obtained after reposition in both groups

\begin{tabular}{lcccc}
\hline & Height $(\mathrm{mm})$ & Tilt $(\mathrm{deg})$ & Inclination $(\mathrm{mm})$ & Variance $(\mathrm{deg})$ \\
\hline Conservative & 10.3 & 1.9 & 18.6 & -1.2 \\
\hline Operative & 10.2 & 6.5 & 16.9 & -0.5 \\
\hline
\end{tabular}

Wilcoxon's test was used while examining paired results of conservatively treated patients. According to Bioethical Commission of Poznan University of Science this research was not a medical experiment.

\section{Results}

Analysis revealed greater redisplacement during healing period in conservatively treated patients. Results proved to be significant in radial inclination and ulnar variance. Mechanism and morphology of fracture had an impact on the results. In group with extraarticular fractures (type A in AO classification) differences were clearer and significant in radial height, inclination and palmar tilt. In intraarticular group (type $\mathrm{C}$ in $\mathrm{AO}$ classification) parameters haven't varied so distinctly, and only ulnar variance differed significantly (Figure 3, Table 3).

Comparing redisplacement in conservatively treated patients, which occurred in particular periods of

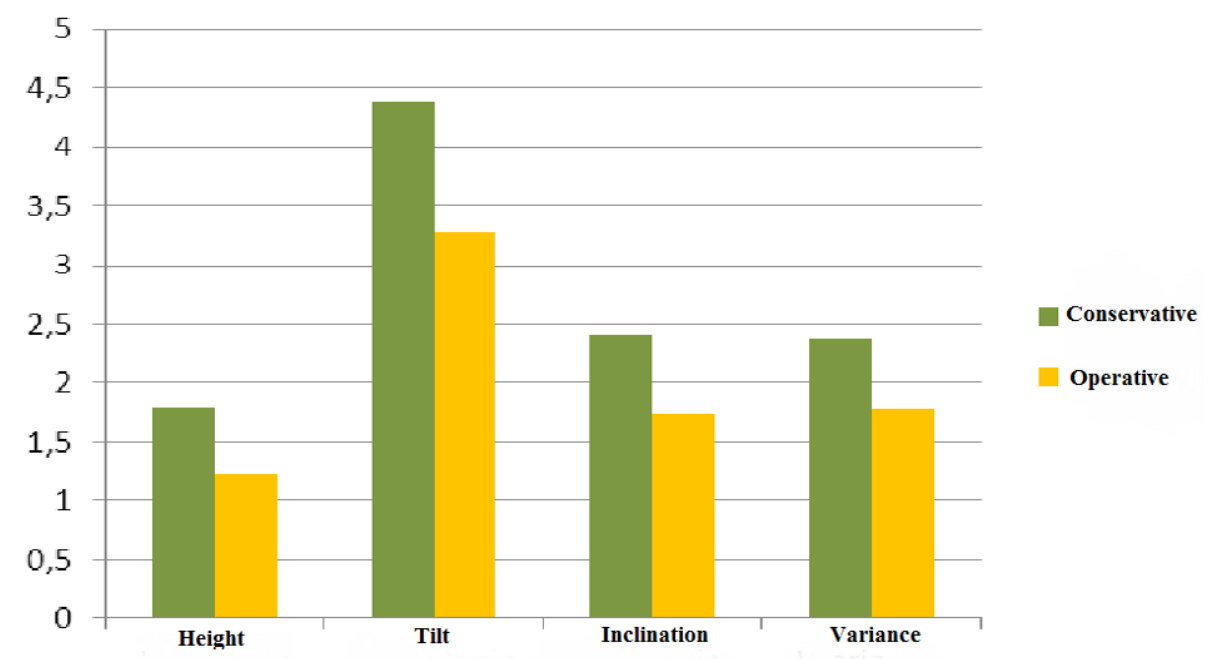

Figure 3. Average redisplacement between reposition and end of treatment in both groups 
Table 3. Average redisplacement between reposition and end of treatment in both groups

\begin{tabular}{lcccc}
\hline & Height $(\mathrm{mm})$ & Tilt $(\mathrm{deg})$ & Inclination $(\mathrm{mm})$ & Variance $(\mathrm{deg})$ \\
\hline Conservative & 1.78 & 4.39 & 2.39 & 2.37 \\
\hline Operative & 1.23 & 3.28 & 1.73 & 1.77 \\
\hline $\mathrm{p}<0.05$ & $\mathrm{~ns}$ & ns & 0.02 & 0.03 \\
\hline
\end{tabular}

immobilization revealed there is no significant difference between average change in radiological parameters in first 7 days and following weeks before cast removing.

Intraarticular fractures (type C) lead to greater redisplacement than extraarticular fractures (type A) irrespective to method of treatment. Differences were significant in ulnar variation (conservative treatment), radial inclination and palmar tilt (operative treatment).

\section{Discussion}

Among several radiological parameters describing three-dimensional structure of distal radius radial height, radial inclination, ulnar variance and palmar tilt were chosen. Posttraumatic changes of these parameters have greatest impact on biomechanical conditions and range of motion in wrist. AO classification was used to describe morphology of fractures, as it is one of the most accurate and enables to assess type of fracture in relatively objective manner by different doctors [11, 12]. Many physicians point out necessity of close control of healing process and fast diagnosing of redisplacement. However I haven't met uniform protocol of follow-up, 7 days intervals are considered as sufficient [13]. For patient's safety reason it would be appropriate to control fragments alignment every 7 days in first three weeks, when fracture consolidates [13]. Unfortunately, due to limited access to OP clinic X-ray scans were obtained after 7 days and 4 to 6 weeks in patients immobilized and after 6 weeks in operated patients. After 6 weeks risk of redisplacement is very low [14].

Examined group of elderly people, with average age close to 70 , varied distinctly in respect of general condition, physical fitness, intellectual abilities and motivation to obey recommendation, as well as ability to finance further rehabilitation. For this reasons objective evaluation of functional results of treatment was impossible. Radically different needs and expectations make there is often no correlation between patient's satisfaction and objective, radiological outcome [15]. In this group there is no relation between anatomic reposition and functional results $[16,17]$. Furthermore comparing dexterity of patients operated with volar plate, results showed, that older patients need almost 6 months longer rehabilitation to achieve progress similar to the young ones [18].

Analysis revealed greater redisplacement during healing period in conservatively treated patients in all parameters, but only radial inclination ulnar variance were significant. We have to consider different mechanism of trauma, leading to varied types of fracture. In type A fractures we often meet severe dorsal displacement and compression of dorsal cortex. In this group results were significantly better in all parameters except ulnar variance. However open reduction with internal fixation allows more accurate reposition, in type $\mathrm{C}$ fractures differences were not that clear. Main goal of this examination was to assess stability on reposition, not the quality. Therefore patients with worse reposition were less prone to redisplacement.

Two plates had to be removed, due to too long screws irritating extensor policis longus tendon. Luckily we avoided rupture of the tendon. No serious complication or infections were noticed in examined group.

Although conservative treatment should be reserved for nondisplaced and stable fractures, sometimes it must be conducted in patient, that don't agree for operation or are disqualified due to general condition. Each patient immobilized in cast underwent radiological control after 7 days. Immediate revealing of redisplacement and operative treatment gives better results than delayed osteotomies [21]. Evaluating redisplacement taking place in first 7 days and following weeks I found they don't vary significantly. Therefore we may implicate patients with borderline alignment of fragments should be qualified for surgery faster. Another closed reposition in these patients is difficult, and gives acceptable results in only $1 / 3$ of all fractures [22].

\section{Conclusions}

Open reduction and internal fixation leads to minor redisplacement comparing to closed reduction and cast immobilization. Type of fracture has great impact on further changes of radiological parameters. 
Intraarticular fractures (type C) lead to greater redisplacement than extraarticular fractures (type A) irrespective of method of treatment.

Redisplacement in operatively treated patients in first 7 days and following 4 to 6 weeks don't vary significantly.

\section{Acknowledgements}

Author would like to thank prof. Leszek Romanowski for guidance and priceless advices, as well as doctors from Department of Orthopedics, General Hospital in Szczecinek for teaching hard orthopedic craft.

\section{Conflict of interest statement}

The authors declare that there is no conflict of interest in the authorship or publication of contribution.

\section{Funding sources}

There are no sources of funding to declare.

\section{References}

1. Ark J, Jupiter JB. The rationale for precise management of distal radius fractures. Orthop Clin North Am. 1993;24:205-10.

2. Robertsson GO, Jonsson GT, Sigurjonsson K. Epidemiology of distal radius fractures in Iceland in 1985. Acta Orthop Scand. 1990;61:457-9.

3. Kakarlapudi TK, Santini A, Shahane SA, et al. The cost of treatment of distal radial fractures. Injury. 2000;31:229-32.

4. McMurtry RY, Jupiter JB, Browner BD Fractures of the distal radius. In Skeletal Trauma: Fractures, Dislocations, Ligamentous Injuries. 1997.

5. Andreas P. Diamantopoulos Gudrun Rohde The Epidemiology of Low- and High-Energy Distal Radius Fracture in Middle-Aged and Elderly Men and Women.

6. João Carlos Belloti. Marcel Jun Sugawara Tamaoki. Carlos Eduardo da Silveira Franciozi Are distal radius fracture classifications reproducible? Intra and interobserver agreementepartment of Orthopedics and Traumatology, Universidade Federal de São Paulo.

7. Nicola Toufik, Torklus Detlef Atlas dostępów operacyjnych $w$ chirurgii ortopedycznej i traumatologii. U\&P, 2009.

8. Jupiter J, Ring D. Surgical treatment of redisplaced fractures of the distal radius in patients older than 60 years. J Hand Surg. 2002;27:714-724.

9. Jupiter JB, Ring D. Open reduction and internal fixation of fractures of the distal radius. American Academy of Orthopedic Surgeons Monograph Series. 1998.

10. Kreder HJ, Hanel DP, McKee MD, Jupiter JB, McGillivary G, Swiontkowski MF. X-ray film measurements for healed distal radius fractures. J Hand Surg [Am]. 1996;21:31-3.
11. Kreder HJ, Hanel DP. Consistency of AO fracture Classification for the distal radius. From Sunnybrook Health Science Center and the University of Toronto, Canada.

12. Andersen GR, Rasmussen J-B, Dahl B, Solgaard S. Older's classification of Colles' fractures: good intraobserver and interobserver reproducibility in 185 cases. Acta Orthop Scand. 1991;62:463.

13. David L Nelson, A Lee Osterman, Harris Gellman Distal fractures of the radius Treatment \& Management. Medscape.

14. Maureen Leahy, Predicting displacement after closed treatement of distal radius fracture. www.aaos.org/news/ aaosnow.

15. Wakefield AE, McQueen MM. The role of physiotherapy and clinical predictors of outcome after fracture of the distal radius. J Bone Joint Surg Br. 2000;82:972-976.

16. Horne JG, Devane P, Purdie G. A prospective randomized trial of external fixation and plaster cast immobilization in the treatment of distal radial fractures. J Orthop Trauma. 1990

17. Howard PW, Stewart HD, Hind RE, Burke FD. External fixation or plaster for severely displaced comminuted Colles' fractures? A prospective study of anatomical and functional results. J Bone Joint Surg Br. 1989.

18. Chung KC, Squitieri L, Kim HM. Comparative outcomes study using the volar locking plating system for distal radius fractures in both young adults and adults older than 60 years. J Hand Surg Am. 2008 Jul-Aug;33(6):809-819.

19. Viegas SF, Tencer AF, Cantrell J, Chang M, Clegg P, Hicks C, O'Meara C, and Williamson JB. Load Transfer Characteristics of the Wrist: Part II. Perilunate Instability. Journal of Hand Surgery. 1987.

20. Rikli DA, Honigmann P, Babst R, Cristalli A, Morlock M, Mittlmeier T. Intraarticular pressure measurement in the radioulnocarpal joint using a novel sensor: in vitro and in vivo results. 2007.

21. Jupiter J, Ring D. A comparison of early and late reconstruction of malunited fractures of the distal end of the radius. J Bone Joint Surg Am. 1996;78(5):739-748.

22. Leung F., Ozkan M.., Chow S. P. Conservative treatment of intra articular fractures of the distal radius-factors affecting functional outcome. Hand Surg. 2000;5(2):145-153.

Acceptance for editing: 2015-02-10 Acceptance for publication: 2015-03-30

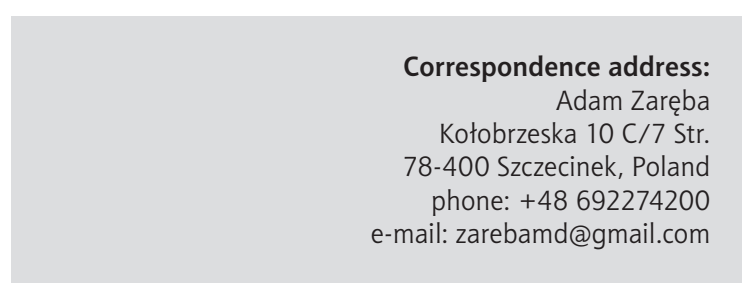

